\title{
DESENVOLVIMENTO DE UM SOFTWARE PARA DIMENSIONAMENTO DE FUNDAÇÕES DO TIPO TUBULÃO PARA TORRES DE LINHAS DE TRANSMISSÃO
}

\section{DEVELOPMENT OF A SOFTWARE FOR SIZING TUBE TYPE FOUNDATIONS FOR TRANSMISSION LINE TOWERS}

\author{
R. O. MARINHO ${ }^{1 *}$, M. D. C. FERREIRA ${ }^{2}$, J. M. A. OLIVEIRA ${ }^{3}$ e G. L. SILVA ${ }^{4}$
}

${ }^{1}$ Instituto Federal de Educação, Ciência e Tecnologia da Paraíba, Coordenação de Edificações, Guarabira, Paraíba, Brasil

${ }^{2}$ Universidade Tecnológica Federal do Paraná, Coordenação de Engenharia Civil, Londrina, Paraná, Brasil

${ }^{3}$ Universidade do Estado do Rio Grande do Norte, Departamento de Matemática, Patu, Rio Grande do Norte, Brasil ${ }^{4}$ Universidade Federal Rural do Semi-Árido, Departamento de Engenharia, Caraúbas, Rio Grande do Norte, Brasil

${ }^{*}$ Renata de Oliveira Marinho. Instituto Federal de Educação, Ciência e Tecnologia da Paraíba, Guarabira, Paraíba, Brasil, Telefone: +55 83 987296287 endereço de e-mail: renata.oliveira@ifpb.edu.br (R. O. Marinho).

\begin{tabular}{l} 
A R T I C L E I N F O \\
\hline Article history: \\
Received 2020-09-29 \\
Accepted 2020-12-12 \\
Available online 2020-12-12 \\
pa l a v r a $s$ - ch a v e \\
Projeto de estruturas \\
Torres autoportantes \\
Linhas de Transmissão \\
Python \\
ke yw or $d s$ \\
Structural Project \\
Self-support tower \\
Transmission lines \\
Python
\end{tabular}

\begin{abstract}
A B S T R A C T
Electricity is an indispensable resource for modern life. In this sense, since the transmission lines are responsible for transmitting energy or signals over long distances and in view of their importance in the context of the electric sector in Brazil, it is essential to develop a welldesigned project for these buildings, ranging from its foundation to elements that compose them. The present work presents the development of a software to assist in the design of the elements of foundations of self-supporting towers of transmission lines according to the standards in force in the country using the Python programming language. To validate the results obtained by the implemented software, an application example is presented with the results compared to those obtained from an analytical solution.
\end{abstract}

\begin{abstract}
R E S U M O
A energia elétrica é um recurso indispensável à vida moderna. Neste sentido, sendo as linhas de transmissão as responsáveis por transmitirem energia ou sinais a longas distâncias e tendo em vista sua importância no contexto do setor elétrico do Brasil, se torna primordial desenvolver um projeto bem elaborado destas construções, que vai desde sua fundação até os elementos que as compõe. O presente trabalho apresenta o desenvolvimento de um software para auxiliar no projeto dos elementos de fundações de torres autoportantes de linhas de transmissão conforme as normas vigentes no país utilizando a linguagem de programação Python. Para validar os resultados obtidos pelo software implementado apresenta-se um exemplo de aplicação com os resultados comparados aos obtidos de uma solução analítica.
\end{abstract}




\section{INTRODUÇ̃̃̃O}

Devido ao grande crescimento urbano, há a necessidade da construção de grandes centrais de produção de energia elétrica para suprir a necessidade de tal desenvolvimento, onde se torna fundamental o uso de uma extensa rede de transmissão de energia. A construção de linhas de transporte de energia depende de fatores, como a topografia do terreno, a composição geológica do mesmo afetando o tipo de fundação que será utilizada nas superestruturas (MENEZES, 2015).

As linhas de transmissão são compostas por torres metálicas treliçadas, cabos, para-raios e isoladores que trilham longos percursos. No decorrer dos trajetos, as torres são implantadas em solos de origem e propriedades geotécnicas variadas, como já mencionado. E tendo em vista sua importância no contexto do setor elétrico do país, é primordial desenvolver um bom projeto destas construções desde sua fundação até os elementos que compõe sua estrutura em si, para que as mesmas resistam aos esforços submetidos e desempenhe seu papel de forma segura (AMARAL, 2015).

Até meados do século XIX, os projetos estruturais eram desenvolvidos manualmente e impossibilitava análises mais realistas dos carregamentos e comportamentos das torres. Por volta de 1970, iniciou-se a popularização dos computadores e foi introduzido o cálculo eletrônico na engenharia estrutural, possibilitando as mais variadas combinações na relação altura/carregamentos. Com isso, as reações nas fundações ficaram mais reais e seus valores mais concretos (CHAVES, 2004).

Nesse contexto, o presente trabalho apresenta o desenvolvimento de um software para auxiliar no projeto do elemento de fundação do tipo tubulão para torres autoportantes de linhas de transmissão conforme as normas vigentes no país e utilizando a linguagem de programação Python.

\section{MATERIAIS E MÉTODOS}

\subsection{Parâmetros geométricos do tubulão}

Determinação do diâmetro da base: Conforme Chaves (2004), o diâmetro da base pode ser determinado por tentativas de maneira a garantir que as tensões horizontais estejam dentro dos valores admissíveis. O mesmo também pode ser determinado a partir da tensão admissível do solo. Albieiro e Cintra (1996) recomendam para a tensão admissível, para qualquer tipo de solo brasileiro, na prática de projetos a utilização da Equação 1.

$\sigma_{a d m}=\frac{N S P T}{5}$

Com isso, pode-se determinar o diâmetro da base a partir da Equação 2, apresentada por Alonso (2010).

$D_{b}=\sqrt{\frac{4 P}{\sigma_{a d m} . \pi}}$ onde P é a força vertical aplicada na fundação, [MN], e $\sigma_{\text {adm }}$ é a tensão admissível do solo determinada pela equação 1, [MPa].

Determinação do diâmetro do fuste: A Equação 3 determina a tensão admissível do concreto de acordo com a NBR 6122:1996, no qual os parâmetros $\gamma_{c}$ e $\gamma_{f}$ possuem valores de 1,6 e 1,4 , respectivamente.

$\sigma_{\text {conc }}=\frac{0.85 \cdot f_{c k}}{\gamma_{c} \cdot \gamma_{f}}$

onde fck é a resistência característica do Concreto à compressão, [MPa].

Segue na sequência a Equação 4 para a determinação do diâmetro do fuste.

$D_{f}=\sqrt{\frac{4 P}{\sigma_{\text {conc }} \pi}}$

onde $\mathrm{P}$ é a força vertical aplicada na fundação, [MN], e $\sigma_{\text {conc é a }}$ tensão admissível do concreto, [MPa], determinada pela equação 3 .

Determinação da área da base e da área do fuste: $\mathrm{O}$ tubulão em estudo possui seção circular em seu fuste e em sua base, desse modo, pode-se determinar a área da base $\left(\mathrm{A}_{\mathrm{b}}\right)$ e a área do fuste $\left(\mathrm{A}_{\mathrm{f}}\right)$ de acordo com as Equações 5 e 6 , respectivamente.

$A_{b}=\frac{\pi \cdot D_{b}^{2}}{4}$
$A_{f}=\frac{\pi \cdot D_{f}^{2}}{4}$

Determinação da altura do cone: A altura do cone $(\mathrm{H})$ é determinada a partir do diâmetro da base e do fuste e do ângulo de inclinação com a horizontal. Conforme Velloso e Lopes (2010), os alargamentos da base são executados de forma que não necessite de armadura e para isso, adota-se um ângulo de $60^{\circ}$ com a horizontal. No qual, essa altura não deve ultrapassar 2 m, de acordo com a NBR 6122:1996. Assim, a equação 7 determina esse parâmetro.

$H=\frac{\left(D_{b}-D_{f}\right) \cdot \operatorname{tg} 60^{\circ}}{2} \leq 2 m$

Determinação do volume da fundação: De acordo com Alonso (2010), o volume da base pode ser calculado como a soma do volume de um cilindro de $20 \mathrm{~cm}$ e um "tronco" de cone com altura (H-20). A Equação 8 descreve essa afirmação.

$V_{b}=0,2 \cdot A_{b}+\left(\frac{H-0,2}{3}\right)\left(A_{b}+A_{f}+\sqrt{A_{b} \cdot A_{f}}\right)$

O volume total é determinado pela soma do volume da base com o volume do fuste, conforme a equação 9 . 


$$
V_{t}=V_{b}+\left(\pi \cdot r^{2} \cdot h_{f}\right)
$$

onde $\mathrm{r}$ e $\mathrm{h}_{\mathrm{f}}$ são respectivamente o raio, [m], e a altura do fuste, [m].

Determinação da área de aço mínima em um tubulão: De acordo com a NBR 6122:1996, a taxa de aço nos tubulões não deve ser inferior a $0,5 \%$ da seção necessária, com isso temos a equação 10 .

$A_{s}=0,005 .\left(\frac{\pi \cdot D_{f}^{2}}{4}\right)$

\subsection{Procedimento de cálculo geotécnico e estrutural do tipo tubulão}

A Figura 1 mostra um esquemático do tubulão com base alargada e suas respectivas dimensões.

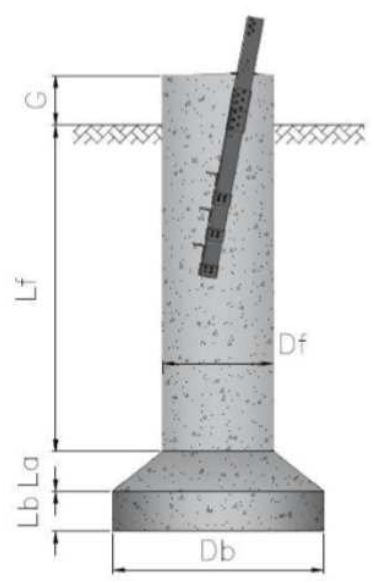

Figura 1 - Esquemático do tubulão com base alargada (AMARAL, 2015).

Verificação à compressão: A tensão atuante de cálculo deve ser menor ou igual a tensão admissível do solo, conforme descrito na Equação 11.

$q_{s}=\frac{P+G_{t u b}-A_{l} \cdot f_{a d m}}{A_{b}} \leq q_{a d m}$

onde qadm é calculado a partir da Equação 12.

$q_{a d m}=\frac{1}{3}\left(c \cdot N_{c} \cdot S_{c}+0,5 \cdot \gamma \cdot B \cdot N_{\gamma} \cdot S_{\gamma}+q \cdot N_{q} \cdot S_{q}\right)$

Verificação ao arrancamento: Para análise ao arrancamento, utilizou-se o método do cone invertido descrito no item 3.5.2, e que pode ser observado na Equação 13.

$G_{t u b}+P_{s} \geq F_{a} . F S$

onde $\mathrm{F}_{\mathrm{a}}$ é a força de arrancamento, $[\mathrm{kN}]$, e FS é o fator de segurança atribuído à estrutura.

Para determinação do volume de terra, segue-se a equação 14 e a equação 15, observando os parâmetros na Figura 2.

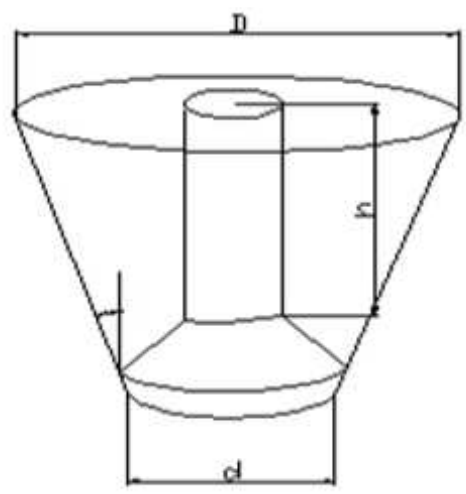

Figura 2 - Parâmetros para o método do cone (QUENTAL, 2008).

$D=d+2 . h \cdot \operatorname{tg} \alpha$

$V_{t}=\left(\frac{\pi \cdot h}{12}\right) \cdot\left(D^{2}+D \cdot d+d^{2}\right)-V_{f}$

onde $\mathrm{V}_{\mathrm{t}}$ é o volume de terra, [m3], $\mathrm{V}_{\mathrm{f}}$ é o volume da fundação (equivalente ao volume de concreto), $\left[\mathrm{m}^{3}\right]$, $\mathrm{D}$ é o diâmetro maior do tronco de cone, $[\mathrm{m}], \mathrm{d}$ é o diâmetro da base da fundação do tronco de cone (equivalente ao $\mathrm{D}_{\mathrm{b}}$ ), $[\mathrm{m}], \alpha$ é o ângulo de inclinação $\left(20^{\circ}\right.$ para areias e $30^{\circ}$ para argilas), e h é a altura do fuste da fundação, $[\mathrm{m}]$.

Cálculo Estrutural da Fundação: A armadura irá resistir os esforços de flexo-compressão e flexo-tração devido aos esforços verticais e horizontais aplicados na fundação, onde essa armadura é distribuída de forma simétrica ao longo do fuste. Para determinação da hipótese que exige maior taxa de armadura

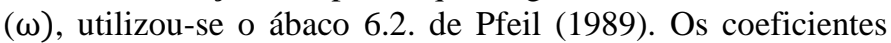
utilizados como entrada são o $v$ e $\mu$, a determinação dos mesmos dependem das equações 16 e 17.

$v=\frac{N_{d}}{f_{c} \cdot D_{f}}$

$\mu=v \cdot\left(\frac{e}{D_{f}}\right)$

onde $e$ representa a excentricidade sendo calculada pela equação 18.

$e=\frac{M_{d}}{N_{d}}$

Temos que, $\mathrm{M}_{\mathrm{d}}$ é o momento de cálculo e é definido pela equação 19 .

$M_{d}=H \cdot\left(G+0,545 \cdot \sqrt{\frac{H}{\gamma+D_{f} \cdot \operatorname{tg}^{2}\left(45+\frac{\varphi}{2}\right)}}\right)$

onde $\mathrm{H}$ é a força horizontal aplicada na fundação, $[\mathrm{kN}]$, $\mathrm{G}$ é o afloramento do tubulão, $[\mathrm{m}], \gamma$ é o peso específico efetivo do solo, $\left[\mathrm{KN} / \mathrm{m}^{3}\right]$, e é o ângulo de atrito do solo, $\left[^{\circ}\right]$. 
Determinação da área de aço da armadura longitudinal do tubulão: A área de aço é determinada pela NBR 6118:2014 pela equação 20 , apresentada a seguir, onde a mesma depende do $\rho$ que é determinado seguindo a equação 21 .

$$
\begin{aligned}
& A_{s}=\rho \cdot\left(\frac{\pi \cdot D_{f}^{2}}{4}\right) \\
& \rho=\omega \cdot\left(\frac{f_{c}}{f_{s}}\right)
\end{aligned}
$$

onde $\omega$ é a taxa de aço determinada pelo ábaco 6.2 de Pfeil (1989), no qual neste trabalho o arranjo foi de 18 barras.

Determinação da área de aço da armadura transversal do tubulão: O diâmetro da armadura transversal é definido pela NBR 6118:2014 no item 18.4.3., onde o mesmo prescreve a Equação 22.

$$
\phi_{w} \geq\left\{\begin{array}{l}
5 m m \\
\frac{\phi_{l}}{4}
\end{array}\right.
$$

Determinação do espaçamento da armadura transversal: O espaçamento da armadura transversal é definido pela NBR 6118:2014 no item 18.4.3, e detalhado na Equação 23, sendo que no presente trabalho, utilizou-se CA 25 para a armadura transversal.

$$
S_{w} \leq\left\{\begin{array}{c}
200 \mathrm{~mm} \\
D_{f} \\
24 . C A 25
\end{array}\right.
$$

Verificação à compressão diagonal e da armadura transversal: As verificações da seção transversal do fuste são feitas utilizando-se o modelo I de cálculo, prevista pela NBR6118:2014, no item 17.4.2.2. O modelo I admite diagonais de compressão inclinadas de $\theta=45^{\circ}$ em relação ao eixo longitudinal do elemento estrutural. Para que haja garantia de segurança da estrutura mediante os esforços transversais, devese respeitar, simultaneamente, as duas seguintes condições (24 e 25).

$$
\begin{aligned}
& V_{s d} \leq V_{r d} \\
& V_{s d} \leq V_{c}+V_{s w}
\end{aligned}
$$

\subsection{Visual Studio Code}

O programa utilizado para desenvolvimento do software foi o Visual Studio Code, em que o mesmo consiste, basicamente, em um editor de código-fonte. A partir dele, tornase possível a elaboração de projetos, como a criação de programas, geração de bancos de dados e isso, em diversas linguagens de programação, dentre elas, o Python. Para elaboração de todo o programa, fez-se uso de diversas bibliotecas, para representação gráfica e solução de problemas matemáticos, onde destacam-se a math, numpy e a plotly. A Figura 3 apresenta a interface do programa Visual Studio Code.

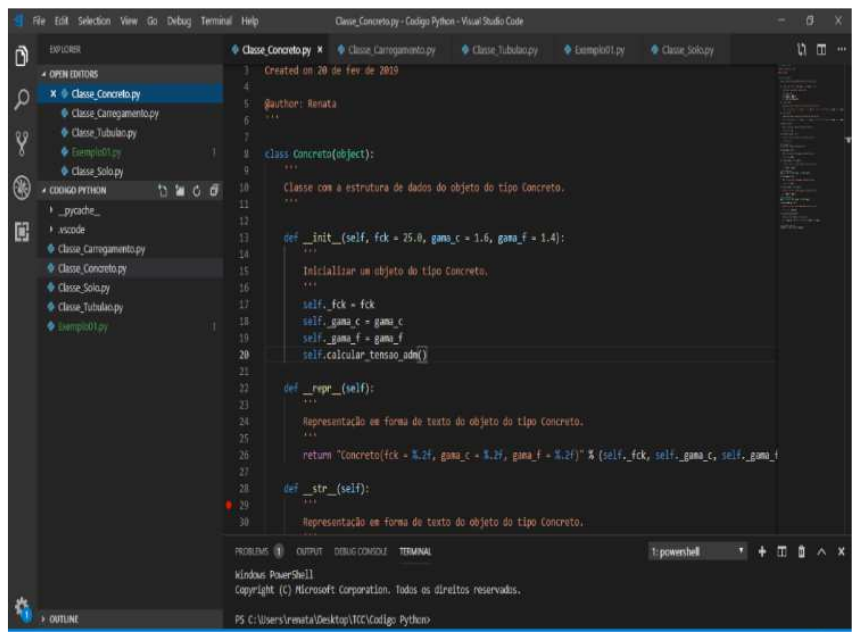

Figura 3 - Interface do programa Visual Studio Code (AUTOR, 2019).

\subsection{Determinação das classes}

A partir das características e efeitos determinados, desenvolveu-se as seguintes classes: carregamento, concreto, solo e tubulão. Na classe de carregamento está inserido os esforços que a torre, provavelmente, será submetida, que são esforços verticais, transversais, longitudinal e horizontal. Onde, o valor do esforço vertical, transversal e longitudinal são valores atribuídos, já o esforço horizontal é o vetor resultante da força longitudinal e transversal. A figura 4 apresenta o diagrama da classe carregamento.

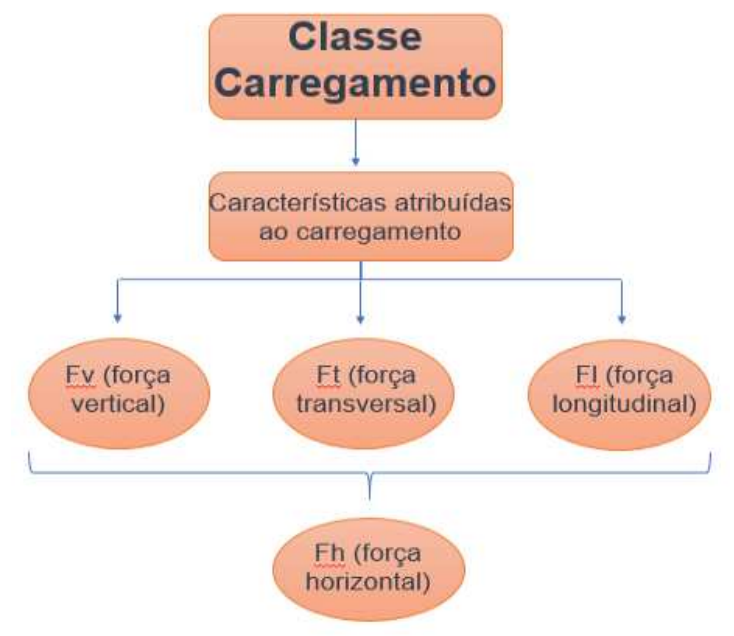

Figura 4 - Diagrama da classe carregamento (AUTOR, 2019).

$\mathrm{Na}$ classe concreto atribuiu-se os parâmetros de $\mathrm{f}_{\mathrm{ck}}, \gamma_{\mathrm{c}} \mathrm{e}$ $\gamma_{\mathrm{f}}$, para em seguida determinar a tensão admissível do concreto e com base nos resultados iniciar a determinação dos parâmetros geométricos do tubulão. A Figura 5 apresenta o diagrama da classe concreto.

Na classe solo, atribuiu-se o valor do NSPT, para em seguida determinar a tensão admissível do solo e com base nos resultados determinar parâmetros geométricos do tubulão. Também se inseriu outros parâmetros de entrada que são característicos ao solo, como: a coesão, o ângulo de atrito representado por $p h i$, o peso específico do solo representado por gama_solo e o tipo de solo onde será implantada a fundação, no 
qual está representado pela variável $t$. A Figura 6 apresenta o diagrama da classe solo.

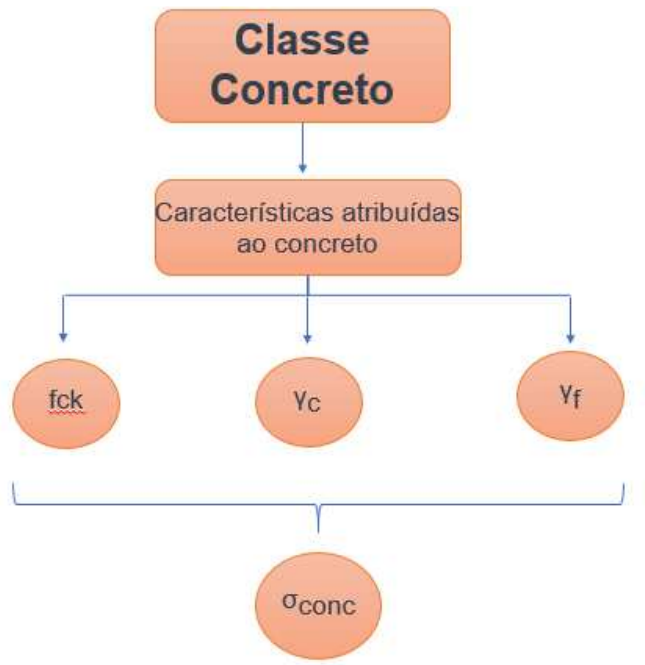

Figura 5 - Diagrama da classe concreto (AUTOR, 2019).

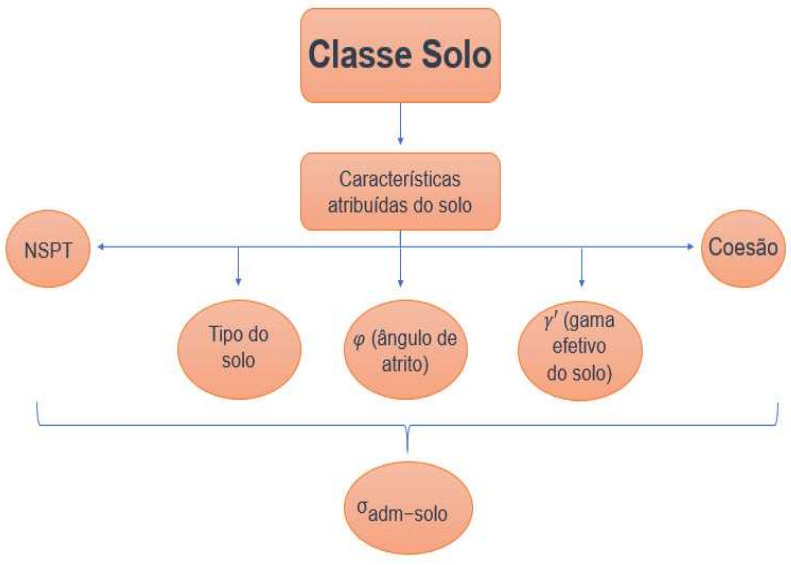

Figura 6 - Diagrama da classe solo (AUTOR, 2019).

Na classe tubulão importou-se as classes anteriores para obter os valores calculados dentro das mesmas e definir todos os parâmetros geométricos, geotécnicos e estruturais citados nos itens, bem como suas verificações que validam a geometria calculada. A Figura 7 apresenta o diagrama da classe tubulão.

Com todos os parâmetros de entrada e de cálculo determinados, seguiu-se para execução do código para o desenho do tubulão com suas respectivas dimensões. Para a implementação do desenho em 2D, utilizou-se a biblioteca plotly, que pode gerar vários tipos de gráficos interativos, dentre outros. Também se utilizou a biblioteca dash para a representação visual em $2 \mathrm{D}$ aliada a plotly. Na sequência, criouse a classe drawTubular para o desenvolvimento do código referente ao desenho, onde seu diagrama está apresentado na Figura 8.

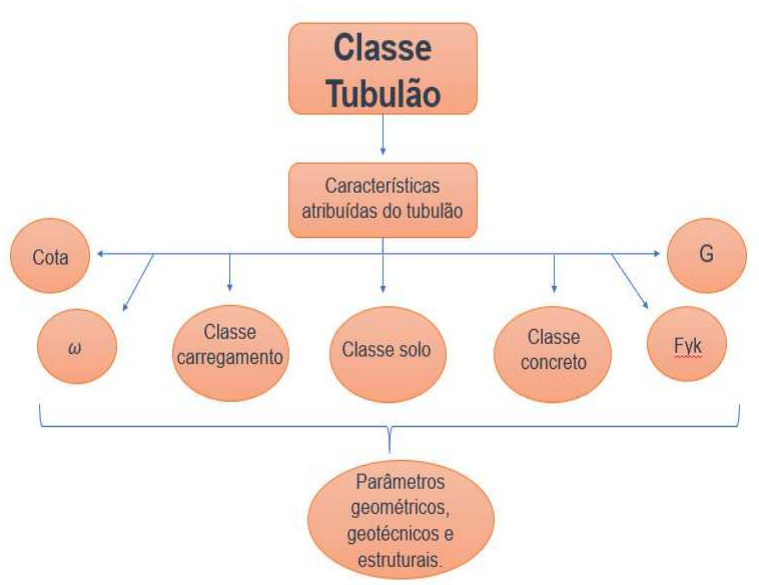

Figura 7 - Diagrama da classe Tubulão (AUTOR, 2019).

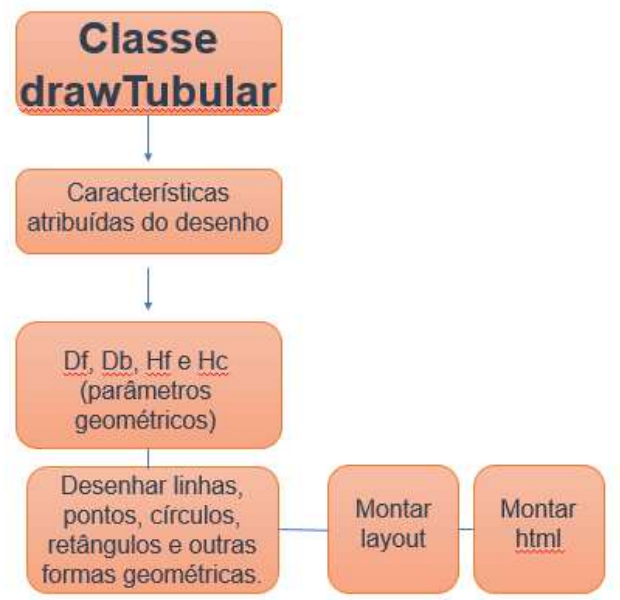

Figura 8 - Diagrama da classe drawTubular (AUTOR, 2019).

\section{RESULTADOS E DISCUSSÃO}

Nesta seção, serão apresentados os resultados do programa desenvolvido. Para avaliar a aplicabilidade desta ferramenta, desenvolveu-se diversos exemplos com resultados comparados e validados com as soluções analíticas obtidas via formulação apresentada no item anterior. Para este trabalho escolheu-se um destes exemplos para demonstrar sua aplicabilidade.

\section{Teste de Aplicação}

Para o exemplo, tem-se os parâmetros de entrada dispostos na Tabela 1 e suas respectivas unidades de entrada no programa.

Com os parâmetros definidos e inseridos no programa, foi possível executá-lo e obter os resultados, que estão descritos na Tabela 2

Na sequência, com o resultado dos parâmetros $v$ e $\mu$, obtidos pelo programa, obteve-se um valor de $\omega=0,1$ no ábaco 6.2. Pfeil (1989), com isso os valores calculados pelo programa estão dispostos na Tabela 3. 
Tabela 1 - Parâmetros de entrada para o exemplo 1.

\begin{tabular}{cc}
\hline Parâmetros de entrada no software \\
Cota / m & 600 \\
Fck / Mpa & 14 \\
NSPT-médio & 15 \\
C (coesão) / kN/m³ & 0 \\
$\varphi$ (ângulo de atrito do solo)/ ${ }^{\circ}$ & 20 \\
$\gamma^{\prime} / \mathrm{kN} / \mathrm{m}^{3}$ & 17 \\
Tipo do solo (t) & areia \\
Fv (força vertical) / kN & 400 \\
Ft (força transversal) / kN & 300 \\
Fl (força longitudinal) / kN & 200 \\
\hline
\end{tabular}

Tabela 2 - Parâmetros geométricos calculados pelo programa - exemplo 1 .

\begin{tabular}{cc|}
\hline \multicolumn{2}{c|}{ Parâmetros Geométricos } \\
$\mathrm{D}_{\mathrm{f}} / \mathrm{cm}$ & 70 \\
$\mathrm{D}_{\mathrm{b}} / \mathrm{cm}$ & 131,55 \\
$\mathrm{Hc} / \mathrm{cm}$ & 53,30 \\
$\mathrm{H}_{\mathrm{f}} / \mathrm{cm}$ & 546,70 \\
$\mathrm{~A}_{\mathrm{b}} / \mathrm{cm}^{2}$ & 13591,57 \\
$\mathrm{~A}_{\mathrm{f}} / \mathrm{cm}^{2}$ & 3848,45 \\
$\mathrm{~V}_{\mathrm{t}} / \mathrm{cm}^{3}$ & 2380538,54 \\
$\mathrm{~A}_{\mathrm{sm}} / \mathrm{cm}^{2}$ & 19,24 \\
$\mathrm{M}_{\mathrm{d}} / \mathrm{kN} \cdot \mathrm{m}$ & 295,19 \\
$e / \mathrm{m}$ & 0,58 \\
$\nu$ & 0,02 \\
$\mu$ & 0,02 \\
\hline
\end{tabular}

Tabela 3 - Parâmetros de cálculo estrutural armadura longitudinal - exemplo 1 .

\begin{tabular}{cc}
\multicolumn{2}{c}{ Armadura Longitudinal } \\
\hline $\mathrm{A}_{\mathrm{st}} / \mathrm{cm}^{2}$ & 19,24 \\
Arranjo das barras $/ \mathrm{mm}$ & $18 \phi 12,5 \mathrm{~mm}$ \\
\hline
\end{tabular}

Foi obtido também o diâmetro da armadura transversal e seu espaçamento seguindo o procedimento contido neste trabalho, em que seus valores podem ser observados na Tabela 4.

Tabela 4 - Parâmetros de cálculo estrutural armadura transversal - exemplo 1.

\begin{tabular}{cc} 
Armadura Transversal & \\
$\phi_{\mathrm{w}} / \mathrm{mm}$ & 5 \\
$\mathrm{~s}_{\mathrm{w}} / \mathrm{cm}$ & 20 \\
\hline
\end{tabular}

As verificações definidas neste trabalho foram executadas de maneira satisfatória e obteve-se os resultados dispostos na Tabela 5.

Tabela 5. Resultados gerados pelo programa para as verificações - exemplo 1.

\begin{tabular}{cc|}
\hline \multicolumn{2}{c}{ Verificação Geotécnicas e Estruturais } \\
\hline Verificação à compressão & $\mathrm{Ok}$ \\
Verificação ao arrancamento & $\mathrm{Ok}$ \\
$\begin{array}{c}\text { Verificação à compressão } \\
\text { diagonal }\end{array}$ & $\mathrm{Ok}$ \\
$\begin{array}{c}\text { Verificação da armadura } \\
\text { transversal }\end{array}$ & $\mathrm{Ok}$ \\
\hline
\end{tabular}

Pode-se observar a partir da Figura 9, que o programa calculou satisfatoriamente os resultados dos parâmetros geométricos para o dimensionamento ao tubulão. Bem como, para determinação dos cálculos geotécnicos e estruturais do mesmo. Com todos os dados estabelecidos, torna-se possível a apresentação do desenho em $2 \mathrm{D}$ produzido pelo programa, facilitando o entendimento e análise dos parâmetros de cálculo. A Figura 9 ainda mostra no final dos resultados obtidos o relatório de execução do programa, no qual é disponibilizado um link que possibilita gerar o desenho em dash de maneira dinâmica e online. A Figura 10 apresenta o desenho em 2D produzido pelo programa.

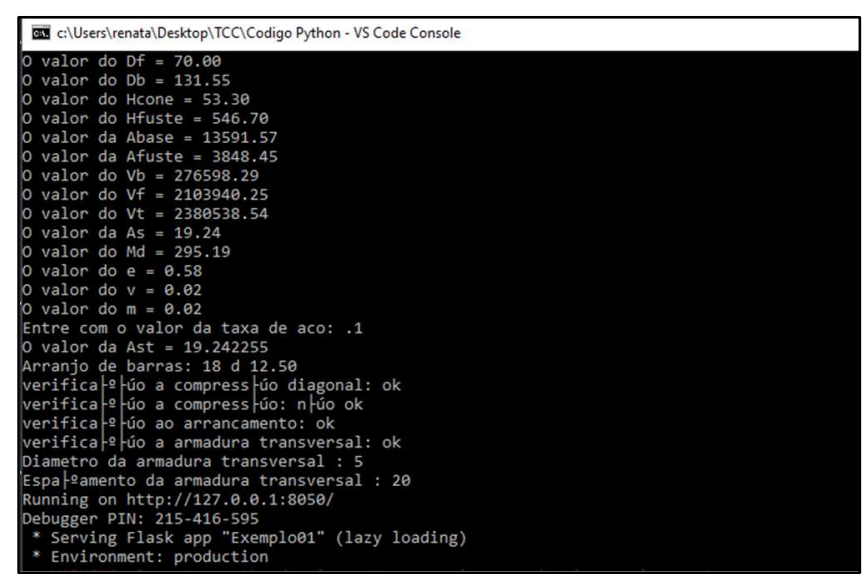

Figura 9 - Resultados gerados com a execução do programa para o exemplo 1 (AUTOR, 2019).

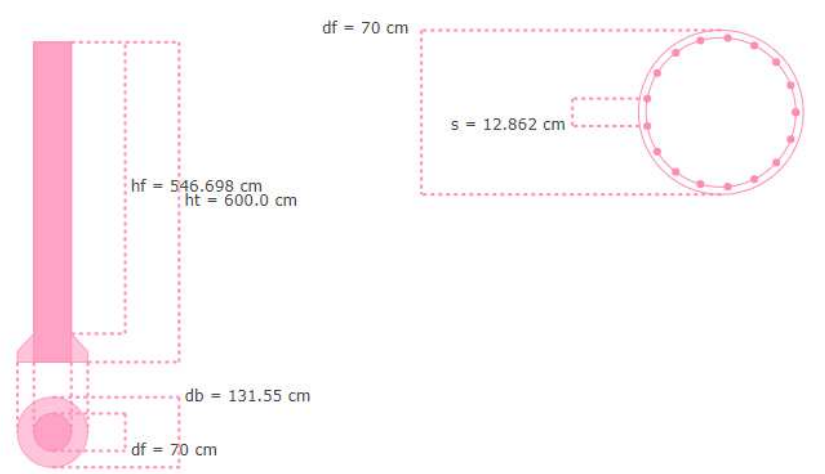

Figura 10 - Resultado do desenho em 2D gerado pelo programa para o exemplo 1. (AUTOR, 2019). 


\section{CONCLUSÕES}

De acordo com os testes e aplicações feitas, foi possível obter informações importantes através dos resultados encontrados. Os resultados encontrados nos diversos exemplos testes, (aqui resumidos a apresentação de um destes testes), e as discussões pertinentes conduziram às seguintes conclusões:

- Foi alcançado de maneira satisfatória a realização do software proposto no presente trabalho, bem como, sua perfeita execução;

- O desenvolvimento dos códigos-fontes para execução do programa foi bem-sucedido;

- O programa foi validado com base na comparação dos resultados obtidos por ele e os obtidos analiticamente pela formulação aqui exposta.

- Desta validação, verificou-se que o software desenvolvido calcula adequadamente todos os parâmetros geométricos necessários para a execução de tubulões;

- O programa também executa adequadamente o cálculo das armaduras longitudinais para o arranjo escolhido no presente trabalho.

De forma geral, o trabalho apresentou o desenvolvimento e a eficiência de um programa para dimensionamento de fundações do tipo tubulões direcionadas para torres de linhas de transmissão garantindo segurança da torre a respeito de todos os esforços que a mesma se encontra submetida.

\section{R E F E R Ê N C I A S}

ALBIERO, J. H.; CINTRA, J. C. A. Análise e projeto de fundações profundas: tubulões caixões. In: Fundações: Teoria e Pratica [S.1: s.n.], 1996.

ALONSO, U. R. Exercícios de Fundações. $2^{\text {a }}$. ed. São Paulo: Edgard Blucher Ltda, 2010.

AMARAL, R. C. Dimensionamento de fundações para torres metálicas de linha de transmissão de energia elétrica. Florianópolis, Santa Catarina, Brasil, 2015.

ASSOCIAÇÃO BRASILEIRA DE NORMAS TÉCNICAS. NBR-6118: Projeto de estrutura de concreto: Procedimento. Rio de Janeiro, 2014. 256 p.

ASSOCIAÇÃO BRASILEIRA DE NORMAS TÉCNICAS. NBR-6122: Projeto e execução de fundações. Rio de Janeiro, 1996. 22 p.

CHAVES, R. A. Fundações de Torres de Linhas de Transmissão e de Telecomunicação. Belo Horizonte, Minas Gerais, Brasil, 2004.

MENEZES, V. P. de. Linhas de transmissão de energia elétrica aspectos técnicos, orçamentários e construtivos. 2015.87 f. TCC (Graduação) - Curso de Engenharia Elétrica, Departamento de Engenharia Elétrica, Universidade Federal do Rio de Janeiro, Escola Politécnica, Rio de Janeiro, 2015.

PFEIL, W. Concreto armado, v. 1, 2 e 3, 5a ed., Rio de Janeiro, Ed. Livros Técnicos e Científicos, 1989

QUENTAL, J. C. (24 de outubro de 2008). Comportamento geomecânico dos solos de fundações das torres da linha de transmissão Recife II/Bongi. 72 Recife, Pernambuco,
Brasil. Acesso em 24 de janeiro de 2019, disponível em: https://repositorio.ufpe.br/bitstream/123456789/5315/1/ arquivo2432_1.pdf.

VELLOSO, D. D. A.; LOPES, F. D. R. Fundações: Critérios de Projeto, Investigação do Subsolo, Fundações Superficiais, Fundações Profundas. São Paulo: Oficina de Textos, v. Completo, 2010. 